\title{
Lactococcus lactis harbouring Ara h 2.02 alleviates allergen-specific Th2-associated responses in sensitized mice
}

\begin{abstract}
Aim: To study the prophylactic effect of recombinant Lactococcus lactis (rLl) harbouring Ara h 2.02 peanut allergen, in sensitized and challenged mice. Methods and results: Ara h 2.02 cDNA was cloned into pNZ8048 for heterologous expression in L. lactis. The purified recombinant allergen showed IgE binding comparable with native Ara h 2. Balb/c mice were fed with either recombinant (rLl), nonrecombinant L. lactis (Ll) or NaHCO3 (Sham) prior to sensitization and challenged with rAra h 2.02, whereas the baseline group was only fed with Ll. Allergen-specific immunoglobulin and splenocyte cytokines responses were determined for each mouse. Mice fed with either Ll or rLl showed significant alleviation of IgE and IgG1 compared to the Sham group. Despite no significant decrease in Th2 (IL-4, IL-13, IL-6) or increase in Th1 (IFN- $\gamma$ ) cytokines, both groups showed lower IL-10 level, while the IL-4 : IFN$\gamma$ ratio was significantly lower for $\mathrm{rLl}$ compared to L1 group. Conclusions: Oral administration of rLl harbouring Ara h 2.02 demonstrated alleviation of Th2-associated responses in allergenchallenged mice and a possible added allergen-specific prophylactic effect. Significance and impact of the study: Ara h 2.02 coupled with the intrinsic properties of probiotic L. lactis as a delivery vehicle can be explored for the development of a commercially scalable vaccine.
\end{abstract}

Keyword: Lactococcus lactis; IgE alleviation; Oral administration; Peanut allergy; Recombinant Ara h 2.02 